\title{
Equivalence of replica and cavity methods for computing spectra of sparse random matrices
}

\author{
František Slanina \\ Institute of Physics, Academy of Sciences of the Czech Republic, \\ Na Slovance 2, CZ-18221 Praha, Czech Republic *
}

(Dated: November 9, 2018)

\begin{abstract}
We show by direct calculation that the replica and cavity methods are exactly equivalent for the spectrum of Erdős-Rényi random graph. We introduce a variational formulation based on the cavity method and use it to find approximate solutions for the density of eigenvalues. We also use this variational method for calculating spectra of sparse covariance matrices.

PACS numbers: 05.40.-a; 89.75.-k; 63.50.Lm
\end{abstract}

\section{INTRODUCTION}

Random matrix theory is a discipline with wide range of physical applications and plenty of beautiful mathematical results [1]. One of the aspects which makes the problem extremely complex is the fact that real physical systems are embedded in three-dimensional Euclidean space. Their Hamiltonian is often a random matrix, but the randomness is constrained in a highly non-trivial way.

The constraints are relatively less severe in the atomic nucleus, where the three-dimensionality of physical space is of secondary importance. Hence the spectacular success of the early works in random matrix theory, due to Wigner [2, 3] and Dyson [4]. On the other hand, the fundamental constraint arising from two-body character of the interaction within the (model of an) atomic nucleus induces several drastic changes [5 9]. Most importantly, the density of states is not a semi-circle, as suggested by Wigner, but rather it follows a Gaussian shape. Therefore, sharp band edges are missing and Lifschitz tails develop. For the current state of the problem, see e. g. the review [10].

Even more complicated situation arises in all random extended systems, like disordered or amorphous semiconductors, where we must take into account the Euclidean constraints. Perhaps the easiest of these constraints is the sparsity of the Hamiltonian matrix, which is due to finite range of interactions. If we forget the even more severe complications due to precise number of spatial dimensions (in reality one, two or three), we are left with the problem of determining the spectrum of a random sparse matrix.

Important breakthrough was achieved using the replica method, which was introduced in the context of random matrices in [11]. Rodgers and Bray, in their classical work [12], solved the problem in the sense that they found an integral equation for a quantity from which the density of states is readily obtained. Unfortunately, that equation still resists all attempts for exact analytic solution.

*Electronic address: slanina@fzu.cz
In [12], two approximative solutions were found. First, in the form of a series expansion, whose leading term coincides with the Wigner semicircle law. Second, using a non-perturbative argument, introduced earlier in [13], the shape of the Lifschitz tails in the density of states was found.

The replica method for treating spectra of sparse matrices was further developed [14 28]. Especially, the variational formulation of the replica equations [19, 20, 27] enabled generating self-consistent approximations, namely the effective medium approximation (EMA), which is analogous to the coherent potential approximation used for electrons in random potential. In these approximations, Lifschitz tails in the spectrum are absent. Further sophistication of the method consists in the single defect approximation (SDA), which obtains the Lifschitz tail in the form of infinite sequence of delta-peaks.

The complexity of the problem becomes evident when we compare these results with the density of states obtained by numerical diagonalization of large sample matrices [27, 29 33]. The Lifschitz tail is smooth, while the bulk of the density of states is the combination of continuous component with a set of delta-peaks. The most marked of these peaks is at the origin, others at eigenvalues $z= \pm 1, \pm \sqrt{2}$ etc. All these structures should emerge from the solution of the Rodgers-Bray integral equation, but EMA, as well as SDA, miss all of them. The set of delta-peaks was studied separately in 20, 32], but a theory which would combine naturally both these peaks and the continuous component is still unavailable.

More recently, spectra of sparse matrices encoding the structure of random graphs were studied successfully using the cavity approach (see e. g. [34]). It is based on the fact that large random graphs are locally isomorphic to trees. This was used e. g. in [35 37] to calculate spectra of adjacency matrix and Laplacian on complex networks. In [35, 36], a "self-consistent" version of SDA was used to obtain asymptotic shape of the Lifschitz tails, which decay as a power law in the case of scale-free networks. In 37] a more sophisticated calculation lead to an integral equation similar to Rodgers and Bray's [12], from which the asymptotics of Lifschitz tails is found. The cavity method provides an easy way [33] to obtain the Wigner 
semicircle law, as well as the Marčenko-Pastur law for spectrum of covariance matrices. It can be also used as an efficient numerical procedure 33], reproducing all peculiarities of the density of states, including Lifschitz tails and delta-peaks. The mathematical justification for the use of the cavity approach can be found in [38].

Very powerful method for computing spectral properties of random matrices is based on supersymmetry and was developed in [39, 40] (see also the review [41] and a recent development in [42]). Initially, the results of replica and supersymmetric methods were found in conflict, which resulted in serious criticism of the replica trick in general [43]. Density of states of sparse random matrices was calculated using supersymmetry [44], leading to an equation which was later [45] shown equivalent to the replica result of [12]. However, the correlation of eigenvalues, which was investigated in [46] using supersymmetry for the case of sparse matrices, was not reproduced correctly in replica method, until the integral over all saddle points was properly taken in [47]. Since then, the replica method regained its reputation as an equivalent alternative to supersymmetric methods. This was further supported by a series of papers [48 50]. Finally, let us only mention the works which approach the density of states by computing exactly the moments [31, 51].

In this paper, we show an alternative method to obtain the Rodgers-Bray integral equation using cavity approach. Therefore, we prove exact equivalence of replica and cavity method in this case, which was previously assumed only on the basis of topological considerations concerning random Erdős-Rényi graphs. Moreover, as an important by-product of this proof, we present a variational formulation of the problem, which serves as useful generator of self-consistent approximations.

\section{PROJECTOR METHOD}

We shall investigate the spectrum of the adjacency matrix $L$ of an Erdős-Rényi random graph with $N$ vertices. Therefore, the probability distribution of the matrix elements factorizes

$$
\pi(L)=\prod_{i<j}\left[\pi_{1}\left(L_{i j}\right) \delta\left(L_{i j}-L_{j i}\right)\right] \prod_{i} \delta\left(L_{i i}\right)
$$

where the probability density for a single off-diagonal element is

$$
\pi_{1}(x)=\left(1-\frac{\mu}{N}\right) \delta(x)+\frac{\mu}{N} \delta(x-1) .
$$

The key ingredient of all subsequent analysis is the resolvent

$$
R(z)=(z-L)^{-1}
$$

and its average $\langle R(z)\rangle$ over disorder, taken with the distribution (1). It contains information on the average density of states (here we assume $z$ on the real axis)

$$
\mathcal{D}(z)=\lim _{\epsilon \rightarrow 0^{+}} \frac{1}{N \pi} \operatorname{Tr}\langle R(z-i \epsilon)\rangle .
$$

In the spirit of the cavity method, we focus on a single vertex, surrounded by the rest of the graph. To calculate the diagonal element of the resolvent on this vertex, we use the projector method, formulated generally in [52]. For a different route which also leads to equivalent results, see [34]. Let us have an arbitrary projector $P$ and its complement $P^{C} \equiv 1-P$. Then, the projected resolvent is 52

$$
P R P=\frac{P}{P(z-L) P-P L P^{C} \frac{P^{C}}{z-L} P^{C} L P} .
$$

We denote the singled-out vertex as $i=0$. Let $P_{0}$ be the projector to this vertex. Furthermore, denote $i=1,2, \ldots, k$ neighbors of the vertex 0 on the graph represented by the matrix $L$ and denote also $P_{i}$ projector to the neighbor $i$. Let us use composite indices for other vertices. If $k_{i}$ is the number of neighbors of $i$, denote $[i, 1],[i, 2], \ldots,\left[i, k_{i}-1\right]$ the neighbors of vertex $i$, except the vertex 0 . The projectors to the second neighbors of 0 will be denoted using these indices, so $P_{i, i^{\prime}}$ is projector on the vertex $\left[i, i^{\prime}\right]$. By analogy, we define the projectors to third, fourth etc. neighbors of 0 . Note that on a general graph, some of the projectors may coincide, due to the presence of cycles.

The cavity approach consists in replacing the graph by a tree, which is locally isomorphic to it, i. e. neglecting all cycles on the graph. Algebraically, it is equivalent to the assumption that the complementary projectors can be written as direct sums of projectors corresponding to separate branches of the tree

$$
\begin{aligned}
P_{0}^{C} & =P_{(1)} \oplus P_{(2)} \oplus \ldots \oplus P_{(k)} \\
P_{(i)} P_{i}^{C} & =P_{(i, 1)} \oplus P_{(i, 2)} \oplus \ldots \oplus P_{\left(i, k_{i}-1\right)} \\
P_{\left(i, i^{\prime}\right)} P_{i, i^{\prime}}^{C} & =P_{\left(i, i^{\prime}, 1\right)} \oplus P_{\left(i, i^{\prime}, 2\right)} \oplus \ldots \oplus P_{\left(i, i^{\prime}, k_{i, i^{\prime}}-1\right)}
\end{aligned}
$$

where $P_{(i)} P_{i}=P_{i}, P_{\left(i, i^{\prime}\right)} P_{i, i^{\prime}}=P_{i, i^{\prime}}$, and so forth.

Using the projectors we define the series of scalar functions related to the resolvent

$$
\begin{aligned}
g(z) & =P_{0} R(z) P_{0} \\
g_{i}(z) & =P_{i} \frac{P_{0}^{C}}{z-L} P_{i} \\
g_{i, i^{\prime}}(z) & =P_{i, i^{\prime}} \frac{P_{(i)} P_{i}^{C}}{z-L} P_{i, i^{\prime}} \\
g_{i, i^{\prime}, i^{\prime \prime}}(z) & =P_{i, i^{\prime}, i^{\prime \prime}} \frac{P_{\left(i, i^{\prime}\right)} P_{i, i^{\prime}}}{z-L} P_{i, i^{\prime}, i^{\prime \prime}}
\end{aligned}
$$

From (5) and the assumptions (6) we have the chain of equations for these functions 


$$
\begin{aligned}
g(z) & =\frac{1}{z-\sum_{i=1}^{k} g_{i}(z)} \\
g_{i}(z) & =\frac{1}{z-\sum_{i^{\prime}=1}^{k_{i}-1} g_{i, i^{\prime}}(z)} \\
g_{i, i^{\prime}}(z) & =\frac{1}{z-\sum_{i^{\prime \prime}=1}^{k_{i, i^{\prime}-1}} g_{i, i^{\prime}, i^{\prime \prime}}(z)}
\end{aligned}
$$

On a random tree, the degrees $k, k_{i}, k_{i, i^{\prime}}$ are random variables and therefore also $g(z), g_{i}(z), g_{i, i^{\prime}}(z)$, etc. are random functions of $z$. To describe their properties, we define their generating functions (dependence on $z$ becomes implicit)

$$
\begin{aligned}
G(\omega) & =\left\langle e^{-\omega g(z)}\right\rangle \\
G_{1}(\omega) & =\left\langle e^{-\omega g_{i}(z)}\right\rangle \\
G_{2}(\omega) & =\left\langle e^{-\omega g_{i, i^{\prime}}(z)}\right\rangle \\
G_{3}(\omega) & =\left\langle e^{-\omega g_{i, i^{\prime}, i^{\prime \prime}}(z)}\right\rangle
\end{aligned}
$$

If the graph in question is the Erdős-Rényi random graph, all the degrees in the corresponding random tree are independent and distributed according to the Poisson distribution $P(k)=e^{-\mu} \mu^{k} / k$ !. The average degree $\mu$ is the only free parameter of this model.

Calculation of the generating functions (9) is facilitated by the integral representation

$$
g(z)=\frac{1}{z-\sum_{i=1}^{k} g_{i}(z)}=\int_{0}^{\infty} e^{-\lambda\left(z-\sum_{i=1}^{k} g_{i}(z)\right)} d \lambda
$$

and similarly for the other $g$ 's. Assuming for the moment that $k$ is fixed, we get, after some algebra, the following relation between $G(\omega)$ and $G_{1}(\omega)$

$$
G(\omega)=1+\sqrt{\omega} \int_{0}^{\infty} \frac{d \lambda}{\sqrt{\lambda}} I_{1}(2 \sqrt{\omega \lambda}) e^{-\lambda z}\left[G_{1}(\lambda)\right]^{k}
$$

Now we take into account the Poisson distribution of degrees, which gives

$$
G(\omega)=1+\sqrt{\omega} \int_{0}^{\infty} \frac{d \lambda}{\sqrt{\lambda}} I_{1}(2 \sqrt{\omega \lambda}) e^{-\lambda z+\mu\left(G_{1}(\lambda)-1\right)} .
$$

Repeating the same steps for further generating functions we get

$$
G_{1}(\omega)=1+\sqrt{\omega} \int_{0}^{\infty} \frac{d \lambda}{\sqrt{\lambda}} I_{1}(2 \sqrt{\omega \lambda}) e^{-\lambda z+\mu\left(G_{2}(\lambda)-1\right)}
$$

Note that the form of the relation between $G$ and $G_{1}$ is the same as between $G_{1}$ and $G_{2}$ and generally between $G_{m}$ and $G_{m+1}$ for any $m>0$. This is due to special property of the Poisson distribution, $k P(k) / \mu=P(k-1)$. For any other distribution this does not hold.

For infinitely large tree we suppose that the generating functions $G_{m}, m=1,2,3, \ldots$ converge to a common limit and we can impose the condition of stationarity $G_{1}(\omega)=$ $G_{2}(\omega)$. Therefore, we define a single function $\gamma(\omega)=$ $G(\omega)-1$, for which we have a closed equation

$$
\gamma(\omega)=\sqrt{\omega} \int_{0}^{\infty} \frac{d \lambda}{\sqrt{\lambda}} I_{1}(2 \sqrt{\omega \lambda}) e^{-\lambda z+\mu \gamma(\lambda)} .
$$

It is strictly equivalent to the Equation (18) in [12] (the Rodgers-Bray equation), which was obtained using the replica method. Hence we conclude that explicit calculation showed equivalence of replica and cavity approaches in the case of Erdős-Rényi graph, which is just the situation in which the Rodgers-Bray equation holds. Note however, that the direct computation we used here would fail if the degree distribution was not Poissonian.

\section{VARIATIONAL PROBLEM}

The key result (14) can be reformulated in a different way, more appropriate for approximate solution. As a first step, we define an auxiliary function $\rho(\omega)=$ $e^{-\omega z+\mu \gamma(\omega)}$. Instead of the single equation (14), we can solve the pair

$$
\begin{aligned}
& \gamma(\omega)=\sqrt{\omega} \int_{0}^{\infty} \frac{d \lambda}{\sqrt{\lambda}} I_{1}(2 \sqrt{\omega \lambda}) \rho(\lambda) \\
& \rho(\omega)=e^{-\omega z+\mu \gamma(\omega)} .
\end{aligned}
$$

Direct solution of (15) is as difficult as solving (14). However, we can find a functional, whose stationary point is just defined by equations (15). We can check explicitly that such functional is

$$
\begin{aligned}
& \mathcal{F}[\gamma, \rho]=-\int_{0}^{\infty} \frac{d \omega}{\omega} \gamma(\omega) \rho(\omega)+ \\
& +\frac{1}{2} \int_{0}^{\infty} \frac{d \omega}{\sqrt{\omega}} \int_{0}^{\infty} \frac{d \lambda}{\sqrt{\lambda}} I_{1}(2 \sqrt{\omega \lambda}) \rho(\omega) \rho(\lambda)+ \\
& \quad+\frac{1}{\mu} \int_{0}^{\infty} \frac{d \omega}{\omega} e^{-\omega z+\mu \gamma(\omega)} .
\end{aligned}
$$

Note that we derived, within the cavity approach, a result which is analogous to the functional obtained in [27] using the replica trick.

The variational formulation of the problem is useful as a generator of approximations. In [20] a variational ansatz was used to derive the density of states in effectivemedium approximation (EMA). Let us see now how it is obtained in our cavity procedure. If we take the exponential ansatz for the auxiliary function $\rho(\omega)$, namely

$$
\rho(\omega)=e^{-\sigma \omega}
$$


all integrals in (16) can be performed explicitly and we can extremalize the functional with respect to $\sigma$ and $\gamma(\omega)$. This way we find the cubic equation

$$
\sigma^{3}-z \sigma^{2}+(\mu-1) \sigma+z=0
$$

It is identical to the equation (23) in 20] obtained by the replica method. The solution can be obtained analytically and the density of states is extracted using the formula

$$
\mathcal{D}(z)=\lim _{\epsilon \rightarrow 0^{+}} \operatorname{Im} \frac{1}{\pi \sigma(z-i \epsilon)} .
$$

We can further improve the calculation by the following trick, which we shall refer as "single-shell approximation" within this paper. We may formally write the pair of equations (15) as a set of four equations

$$
\begin{aligned}
& \gamma(\omega)=\sqrt{\omega} \int_{0}^{\infty} \frac{d \lambda}{\sqrt{\lambda}} I_{1}(2 \sqrt{\omega \lambda}) \rho(\lambda) \\
& \rho(\omega)=e^{-\omega z+\mu \bar{\gamma}(\omega)} \\
& \bar{\gamma}(\omega)=\sqrt{\omega} \int_{0}^{\infty} \frac{d \lambda}{\sqrt{\lambda}} I_{1}(2 \sqrt{\omega \lambda}) \bar{\rho}(\lambda) \\
& \bar{\rho}(\omega)=e^{-\omega z+\mu \gamma(\omega)}
\end{aligned}
$$

These equations can be obtained as a condition of stationarity for the functional

$$
\begin{aligned}
\mathcal{F}_{1}[\gamma, \rho, \bar{\gamma}, \bar{\rho}]=-\int_{0}^{\infty} \frac{d \omega}{\omega}(\gamma(\omega) \bar{\rho}(\omega)+\bar{\gamma}(\omega) \rho(\omega)) \\
+\int_{0}^{\infty} \frac{d \omega}{\sqrt{\omega}} \int_{0}^{\infty} \frac{d \lambda}{\sqrt{\lambda}} I_{1}(2 \sqrt{\omega \lambda}) \rho(\omega) \bar{\rho}(\lambda)+ \\
+\frac{1}{\mu} \int_{0}^{\infty} \frac{d \omega}{\omega} e^{-\omega z}\left(e^{\mu \gamma(\omega)}+e^{\mu} \bar{\gamma}(\omega)\right)
\end{aligned}
$$

If the equations (20) were solved exactly, we would have $\gamma(\omega)=\bar{\gamma}(\omega)$ and $\rho(\omega)=\bar{\rho}(\omega)$. The same would hold also in the case of the effective medium approximation, which amounts taking the ansatz $\rho(\omega)=\bar{\rho}(\omega)=e^{-\sigma \omega}$, so seemingly the set (20) does not bring any advantage over (15). However, relaxing the condition $\rho(\omega)=\bar{\rho}(\omega)$ we can get an improvement in an approximate solution. Indeed, we can take the ansatz

$$
\rho(\omega)=e^{-\sigma \omega}
$$

as in EMA, but allow $\bar{\rho}(\omega)$ adjust itself freely so that $\mathcal{F}_{1}$ is stationary. This way we introduce an error, because $\rho(\omega) \neq \bar{\rho}(\omega)$ and $\gamma(\omega) \neq \bar{\gamma}(\omega)$, but we gain better approximation for the density of states.

After some algebra, we get the following equation for the quantity $\tau=z \sigma$

$$
z^{2}=\mu+\tau+e^{-\mu} \sum_{l=1}^{\infty} \frac{\mu^{l}}{(l-1) !} \frac{l}{\tau-l}
$$

The fact that the equation depends on $z^{2}$ means that the spectrum is symmetric with respect to the point $z=0$. For a general $z$ on the real axis the equation (23) can be easily solved numerically. We find that there are at most two roots with non-zero imaginary parts (complex conjugate to each other). Those values of $z$ for which all solutions are real correspond to gaps in the spectrum. General picture is that there is a very narrow gap around $z=0$, separating two halves of a wide band, containing most of the eigenvalues. We can call this band (not quite precisely) as "bulk" of the density of states.

In the middle of the bulk, there is a $\delta$-function contribution just at $z=0$, whose weight can be found exactly and is equal to $\mathrm{e}^{-\mu}$. On both sides of the bulk, there are series of small side bands separated by gaps. The density of states has therefore the form

$$
\mathcal{D}(z)=\mathrm{e}^{-\mu} \delta(z)+\mathcal{D}_{c}(z)
$$

where $\mathcal{D}_{c}(z)$ is a continuous function. The interpretation of the $\delta$-function is straightforward. It corresponds to single isolated vertices, whose fraction is just equal to $\mathrm{e}^{-\mu}$ and they all contribute with the same eigenvalue 0 .

Some analytical information on the continuous part $\mathcal{D}_{c}(z)$ can be found from approximate solution of the equation (23). For $\mathrm{e}^{-\mu} \ll 1$ we can find approximately the edge of the gap around $z=0$. We get

$$
\mathcal{D}_{c}(z) \simeq \frac{1}{2 \pi z} \sqrt{4 \psi(\mu) z^{2}-\mathrm{e}^{-2 \mu}}
$$

where we denoted

$$
\psi(\mu)=\mathrm{e}^{-\mu} \sum_{l=1}^{\infty} \frac{\mu^{l}}{l ! l}=\mu \mathrm{e}_{2}^{-\mu} F_{2}(1,1 ; 2,2 ; \mu) .
$$

We can see that the gap edge is at $z_{0}=\frac{1}{2} \mathrm{e}^{-\mu} / \sqrt{\psi(\mu)}$.

For the tails, we can calculate analytically the side bands in an approximation which becomes exact for $|z| \rightarrow \infty$. The computation goes as follows. Each of the side bands can be identified with one term in the infinite sum over $l$ in (23). The tails of the spectrum corresponding to large $|z|$ are identified with large $l$. In the crudest approximation, the solution is $\tau \simeq l$. Therefore, we introduce a new variable $\eta$ by $\tau=l+\eta$. So, 23) assumes the form

$$
\begin{aligned}
z^{2}= & \mu+l+\eta+e^{-\mu} \frac{\mu^{l}}{(l-1) !} \frac{l}{\eta}+ \\
& +e^{-\mu} \sum_{\substack{l^{\prime}=1 \\
\left(l^{\prime} \neq l\right)}}^{\infty} \frac{\mu^{l^{\prime}}}{\left(l^{\prime}-1\right) !} \frac{l^{\prime}}{l-l^{\prime}-\eta}
\end{aligned}
$$

For large $l$ we can expand the infinite series in powers of $\eta$ and keep only the lowest terms, so

$$
\begin{gathered}
z^{2}=\mu+\Delta_{l}(\mu)+l+\left(1-\Gamma_{l}(\mu)\right) \eta+ \\
+e^{-\mu} \frac{\mu^{l}}{(l-1) !} \frac{l}{\eta}+O\left(\eta^{2}\right)
\end{gathered}
$$


where

$$
\begin{aligned}
& \Delta_{l}(\mu)=e^{-\mu} \sum_{\substack{l^{\prime}=1 \\
\left(l^{\prime} \neq l\right)}}^{\infty} \frac{\mu^{l^{\prime}}}{\left(l^{\prime}-1\right) !} \frac{l^{\prime}}{l-l^{\prime}} \\
& \Gamma_{l}(\mu)=e^{-\mu} \sum_{\substack{l^{\prime}=1 \\
\left(l^{\prime} \neq l\right)}}^{\infty} \frac{\mu^{l^{\prime}}}{\left(l^{\prime}-1\right) !} \frac{l^{\prime}}{\left(l-l^{\prime}\right)^{2}} .
\end{aligned}
$$

So, for each $l$, large enough, we have two "bubbles" of non-zero density of states. The two bubbles are symmetric to each other with respect to the origin. The "bubbles" are separated by gaps, so each "bubble" has well defined lower and upper edges, $z_{l-}$ and $z_{l+}$, respectively. For large $l$ the approximate form of the "bubble" is given by the solution of a quadratic equation in $\eta$, so

$$
\begin{aligned}
\mathcal{D}_{l}(z) & \simeq \frac{|z|}{\pi}\left[\left(1-\Gamma_{l}(\mu)\right) \frac{\mathrm{e}^{-\mu} l \mu^{l}}{(l-1) !}-\right. \\
& \left.-\left(\frac{z^{2}-\mu-l-\Delta_{l}(\mu)}{2}\right)^{2}\right]^{1 / 2} \times \\
& \times\left[\frac{\mathrm{e}^{-\mu} l \mu^{l}}{(l-1) !}+\left(z^{2}-\mu-l\right) l+\right. \\
& \left.+\left(1-\Gamma_{l}(\mu)\right)(l)^{2}\right]^{-1} .
\end{aligned}
$$

The width of the bubble $z_{l+}-z_{l-}$ approaches zero for $l \rightarrow \infty$. This justifies considering $\eta$ a small parameter in the expansion (28). For large $l$ the "bubbles" have a semi-circle shape and their weight is

$$
W_{l}=\int_{z_{l-}}^{z_{l+}} \mathcal{D}_{l}(z) d z \simeq \frac{1}{2} e^{-\mu} \frac{\mu^{l}}{l !} .
$$

We recognize the Poisson distribution with mean $\mu$. This reflects the Poisson distribution of degrees of the random graph. The factor $1 / 2$ stems from the fact that we have two bubbles for each $l$. The center of the bubble corresponding to $l$ is at $z_{l}=\sqrt{l+\mu+\Delta_{l}(\mu)}$, thus the distance between two successive bubbles is $\Delta z_{l} \simeq\left(4 z_{l}\right)^{-1 / 2}$. Hence we deduce the approximate density of states in the tails, for $|z| \rightarrow \infty$

$$
\mathcal{D}_{\text {tail }}(z) \simeq \frac{\mathrm{e}^{-\mu}|z| \mu^{z^{2}}}{\Gamma\left(z^{2}-1\right)} \simeq \frac{\mathrm{e}^{-\mu}}{\sqrt{2 \pi}}\left(\frac{e \mu}{z^{2}}\right)^{z^{2}} .
$$

This is the shape of the Lifschitz tail, which was already obtained by [12] and [20].

To assess the quality of the approximations used, we compare the results arising from EMA (Eq. (18)), from the single-defect [20, 33], and single-shell (Eq. (23)) approximations with average density of states obtained by numerical diagonalization of sample matrices. In Fig. 1 we can see the spectrum for $\mu=3$ and matrices of

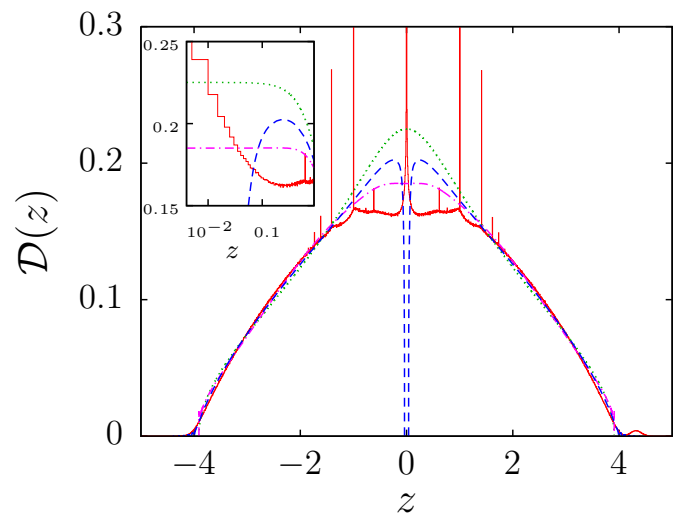

FIG. 1: Density of states for the adjacency matrix of ErdősRényi graph, with average degree $\mu=3$. Full line shows the result of numerical diagonalization of matrix of size $N=1000$, averaged over 75000 random realizations. The dotted line is the result of effective medium approximation, the dot-dashed line id the single-defect approximation, and the dashed line is the single-shell approximation. In the inset, detail of the density of states around the center of the band, plotted in semi-logarithmic scale.

size $N=1000$ averaged over 75000 realizations. We can clearly identify the delta-peaks, as well as the complicated shape of the continuous part of the spectrum near the center of the bulk. Interestingly, both EMA and single-shell approximations are very good if we are neither close to the center nor at the tails of the spectrum. Close to the center, the shape of the density of states is rather complex, as shown in the inset in Fig. 1. There is a shallow depression, followed by a divergence at $z=0$. The form of the singularity at $z=0$ seems to be close to a logarithmic divergence, although the data do not provide a decisive evidence. Neither of the three approximations reproduces this singularity. EMA and SDA are constant around $z=0$, while the single-shell approximation exaggerates the depression around $z=0$ to such extent that a spurious gap is created. This is an artifact of the approximation. However, the delta-peak at the origin is, correctly, present in the single-shell approximation.

Similar comparison was done also at the tail of the density of states. We can see in Fig. 2 a detail of the same data as shown in Fig. 1. Note that, for any finite $N$, the density of states is not mirror-symmetric with respect of the line $z=0$, because the average value of the off-diagonal elements of the matrix $L$ is strictly positive. Only in the limit $N \rightarrow \infty$ the spectrum becomes symmetric. The single largest eigenvalue is split off the rest of the spectrum [53] and the small bump in the positive tail corresponds to this effect. In the limit $N \rightarrow \infty$ this bump would vanish, as the weight of the single largest eigenvalue becomes negligible compared to the rest of the spectrum.

As shown in Fig. 2, we can see that the single-shell approximation is superior to both EMA and SDA in the tail region, from two aspects. First, the spurious band edge 


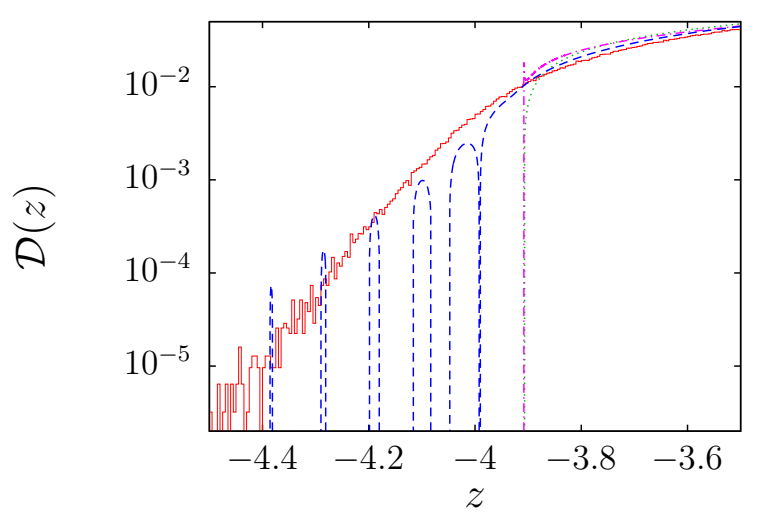

FIG. 2: The detail of the left tail of the density of states shown in Fig. 1. The full line shows the result of numerical diagonalization, the dotted line is the result of effective medium approximation, the dot-dashed line id the single-defect approximation, and the dashed line is the single-shell approximation.

of EMA and SDA is shifted towards larger $|z|$, so that the interval in which $D(z)$ is well reproduced is wider. Second, the single-shell approximation displays non-zero density of states also in some regions of the Lifschitz tails, although, instead of exhibiting a smooth behavior everywhere, the density of states is concentrated in "bubbles". The gaps separating the "bubbles" are again artifacts of the approximation, to the same extent as the sharp band edge is an artifact of EMA and SDA. On the other hand, it is an important improvement over SDA [20]. The delta-peaks of SDA are widened into continuous bands in our approach. In fact, this is to be expected, because the single-shell approximation can be rightly interpreted as a self-consistent version of SDA. Therefore, it should be better than SDA in principle, although this a priori judgement may turn incorrect in practice, as the singleshell approximation is better than SDA sometimes (in the tail) but worse elsewhere (around $z=0$ ).

Finally, let us note that similar "bubbles" at the tails were also seen in approximations derived using replica method by [21] for the Laplacian of a random graph and by [25] for sparse covariance matrices.

\section{COVARIANCE MATRICES}

Another application of the method presented here is investigation of sparse covariance matrices. They can be considered as arising from a bipartite graph where edges connect vertices from the set $A$ with vertices from the set $B$. We denote the size of the sets $N_{A}$ and $N_{B}$, respectively. In the thermodynamic limit, $N_{A} \rightarrow \infty, N_{B} \rightarrow \infty$, we fix the ratio $\alpha=N_{A} / N_{B}$ constant. In the bipartite analog of Erdös-Rényi random graph, the degrees if vertices in $A$ and $B$ follow Poisson distributions with average degree $\mu_{A}$ and $\mu_{B}$, respectively, where $\mu_{B} / \mu_{A}=\alpha$.
The problem has a long history, starting with the work of Marčenko and Pastur [54] and was investigated recently by replica method in [25]. form

The adjacency matrix of the bipartite graph has the

$$
L=\left(\begin{array}{ll}
0 & M^{T} \\
M & 0
\end{array}\right)
$$

where the first block of indices corresponds to set $A$, second block to set $B$. We define the contraction, or covariance, matrix $C_{A}=M^{T} M$, which acts solely in the set $A$ (and similarly $C_{B}=M M^{T}$, which acts solely in the set $B$. The spectra of the matrices $L$, $C_{A}$ and $C_{B}$ are closely related. We define $\mathcal{D}_{A}(z)=$ $\lim _{\epsilon \rightarrow 0^{+}} \operatorname{Im} \sum_{i \in A}\left[(z-i \epsilon-L)^{-1}\right]_{i i} /\left(N_{A} \pi\right)$ the partial density of states of $L$ restricted to the set $A$ and $\mathcal{D}_{C A}(z)=$ $\lim _{\epsilon \rightarrow 0^{+}} \operatorname{Im} \sum_{i \in A}\left[\left(z-i \epsilon-C_{A}\right)^{-1}\right]_{i i} /\left(N_{A} \pi\right)$ the density of states of the correlation matrix $C_{A}$. It can be easily shown that

$$
\mathcal{D}_{C A}(z)=\frac{1}{\sqrt{z}} \mathcal{D}_{A}(\sqrt{z})
$$

This relation remains in force also after averaging over the randomness in the matrix $M$. Therefore, to calculate the average density of states of the covariance matrix $C_{A}$ it is enough to investigate the matrix element $\left\langle\left[(z-L)^{-1}\right]_{i i}\right\rangle$ for any $i \in A$. To this end, we define the generating functions

$$
\begin{aligned}
& \gamma_{A}=\left\langle e^{-\omega\left[(z-L)^{-1}\right]_{i i}}\right\rangle-1 \text { for } i \in A \\
& \gamma_{B}=\left\langle e^{-\omega\left[(z-L)^{-1}\right]_{j j}}\right\rangle-1 \text { for } j \in B .
\end{aligned}
$$

Further procedure follows closely that of the previous section. Finally, we get a set of four coupled equations, very similar to the set we encountered in the single-shell approximation

$$
\begin{aligned}
& \gamma_{A}(\omega)=\sqrt{\omega} \int_{0}^{\infty} \frac{d \lambda}{\sqrt{\lambda}} I_{1}(2 \sqrt{\omega \lambda}) \rho_{B}(\lambda) \\
& \rho_{B}(\omega)=e^{-\omega z+\mu_{A} \gamma_{B}(\omega)} \\
& \gamma_{B}(\omega)=\sqrt{\omega} \int_{0}^{\infty} \frac{d \lambda}{\sqrt{\lambda}} I_{1}(2 \sqrt{\omega \lambda}) \rho_{A}(\lambda) \\
& \rho_{A}(\omega)=e^{-\omega z+\mu_{B} \gamma_{A}(\omega)}
\end{aligned}
$$

We can easily check that the solution of these equations makes the following functional stationary

$$
\begin{aligned}
& \mathcal{F}_{A B}\left[\gamma_{A}, \rho_{A}, \gamma_{B}, \rho_{B}\right]= \\
& -\int_{0}^{\infty} \frac{d \omega}{\omega}\left(\gamma_{A}(\omega) \rho_{A}(\omega)+\gamma_{B}(\omega) \rho_{B}(\omega)\right) \\
& +\int_{0}^{\infty} \frac{d \omega}{\sqrt{\omega}} \int_{0}^{\infty} \frac{d \lambda}{\sqrt{\lambda}} I_{1}(2 \sqrt{\omega \lambda}) \rho_{A}(\omega) \rho_{B}(\lambda)+ \\
& +\int_{0}^{\infty} \frac{d \omega}{\omega} e^{-\omega z}\left(\frac{1}{\mu_{A}} e^{\mu_{A} \gamma_{B}(\omega)}+\frac{1}{\mu_{B}} e^{\mu_{B} \gamma_{A}(\omega)}\right) .
\end{aligned}
$$




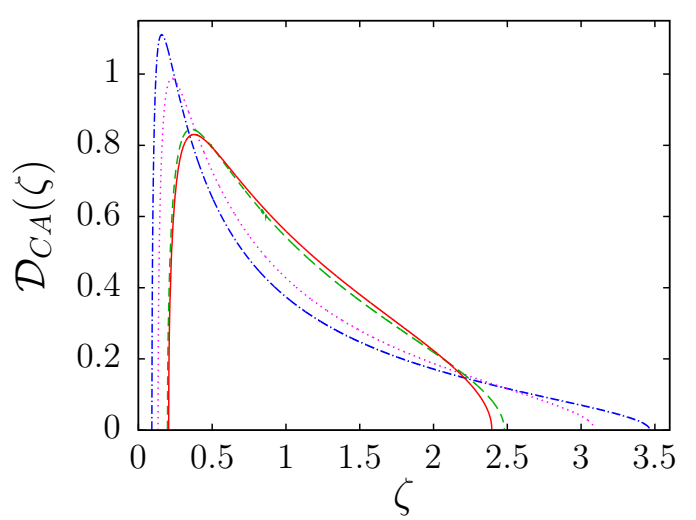

FIG. 3: Density of states for the correlation matrix based on sparse adjacency matrix, for $\alpha=0.3$. The average degree is $\mu_{A}=3$ (dash-dotted line), 5 (dotted line), and 50 (dashed line). The full line is the Marčenko-Pastur density (41), i. e. the limit $\mu_{A} \rightarrow \infty$.

For an approximate solution of the equations (36) we use again a variational ansatz. In analogy with EMA, we assume the following form

$$
\begin{aligned}
& \rho_{A}(\omega)=e^{-\sigma_{A} \omega} \\
& \rho_{B}(\omega)=e^{-\sigma_{B} \omega} .
\end{aligned}
$$

The insertion of (38) in (37) produces finally two uncoupled cubic equations for $\sigma_{A}$ and $\sigma_{B}$. The equation relevant for us is

$$
\begin{aligned}
& z \sigma_{B}^{3}+\left((1-\alpha) \mu_{A}+\alpha-1-z^{2}\right) \sigma_{B}^{2}+ \\
& +\left(\mu_{A} \alpha+1-2 \alpha\right) z \sigma_{B}+z^{2} \alpha=0
\end{aligned}
$$

where we used $\alpha=\mu_{B} / \mu_{A}$. The average density of states for the covariance matrix $C_{A}$ is found considering the first equation of (36) and the relation (34), thus

$$
\mathcal{D}_{C A}(z)=\frac{1}{\pi \sqrt{z}} \lim _{\epsilon \rightarrow 0+} \operatorname{Im} \frac{1}{\sigma_{B}(\sqrt{z}-i \epsilon)} .
$$

The solution can be obtained analytically, but we shall not show the formula here. However, we can check that in the limit $\mu_{A} \rightarrow \infty$ with $\alpha$ and $\zeta=z / \mu_{A}$ fixed we get

$$
\begin{aligned}
\mathcal{D}_{C A}(\zeta) & =\frac{1}{2 \pi \alpha \zeta} \times \\
& \times \sqrt{\left((1+\sqrt{\alpha})^{2}-\zeta\right)\left(\zeta-(1-\sqrt{\alpha})^{2}\right)}
\end{aligned}
$$

which is the Marčenko-Pastur (MP) density of states [54].

In Fig. 3 we show the density of states as function of $\zeta=z / \mu_{A}$ for several values of $\mu_{A}$, as found by solution of (39). We can see that the approach to MP density is rather slow. We found that the difference can be considered small only at about $\mu_{A} \simeq 50$.

\section{CONCLUSIONS}

We considered a random graph of large size $N \rightarrow \infty$ of two types. First, a "classical" Erdős-Rényi graph, and second, a random bipartite graph. We calculated density of eigenvalues of adjacency matrices of these graphs. In the case of the bipartite graph, the final result was the density of states of the covariance matrix, defined by a contraction of the adjacency matrix.

Our contribution to the problem of spectra of sparse random matrices consists in showing that the cavity approach, i. e. approximation of the random graph by a random tree, is exactly equivalent to the calculation by replica method in the thermodynamic limit. Furthermore, we demonstrated how the cavity calculation can be formulated as a variational problem, similar but substantially simpler than the variational formulation arising from the replica method. At minimum, we do not need to consider the possibility of replica-breaking solutions, which are known to exist and contribute to the finite-size corrections [47]. We can interpret it also in the following manner. Since we are working directly with infinite-size system, $N=\infty$, the physics behind the replica-breaking states has no effect.

The variational formulation introduced here is a very practical starting point for approximations. The exponential ansatz leads to results identical to the effectivemedium approximation studied earlier [20]. However, using our variational scheme, the approximation can be easily improved by what we call "single-shell approximation". It produces the Lifschitz tail in the density of states in the form of a series of "bubbles". We are able to calculate the weight and distance of the bubbles. Hence we arrive at average density of states in the tail, which is identical to the old result of Rodgers and Bray [12]. Furthermore, we applied the method also to the spectra of sparse covariance matrices, where we easily derived a formula generalizing the Marčenko-Pastur density of states.

The variational formulation introduced here can be used not only as a generator of approximations, but also as a basis of numerical methods. Indeed, there is no principal obstacle for numerical extremalization of the functional of two variables. This contrasts with the variational methods based on replica trick, where the replica limit $n \rightarrow 0$, involving analytic continuation, must be done after extremalization, whch makes the method numerically unfeasible.

We believe that the method can be applied also for other types of random graphs. We must, however, admit a serious limitation of our method, which is the Poisson distribution of degrees of the graph. Therefore, it is, for example, not applicable directly for graphs with powerlaw degree distribution. We believe that the roots of this limitation lie quite deep. For example, to our best knowledge, there is no replica calculation available for random graphs defined by their degree sequence only. And, on the other side, there are no results from cavity method 
for those random graphs with power-law degree distribution, for which replica calculations do exist, like those of Ref. [23]. The point is, that for Erdős-Rényi graph, it is well established that the local topology is isomorphic to a random tree. For a graph with general degree sequence, non obeying Poisson statistics, this may or may not be true. The question of equivalence or not of replica and cavity methods is intimately related to the question of local isomorphism to a tree, which is rather complex and not solved in general. Hence, a successful treatment of such cases by both replica and cavity method in parallel, would require, very probably, completely novel ideas.

\section{Acknowledgments}

I gladly acknowledge inspiring discussions with J. Mašek. This work was carried out within the project AV0Z10100520 of the Academy of Sciences of the Czech Republic and was supported by the MŠMT of the Czech Republic, grant no. OC09078.
[1] M. L. Mehta, Random matrices (Academic Press, San Diego, 1991).

[2] E. P. Wigner, Ann. Math. 62, 548 (1955).

[3] E. P. Wigner, Ann. Math. 65, 203 (1957).

[4] F. J. Dyson, J. Math. Phys. 3, 140 (1962).

[5] J. B. French and S. S. M. Wong, Phys. Lett. B 33, 449 (1970).

[6] O. Boghias and J. Flores, Phys. Lett. B 34, 261 (1971).

[7] O. Boghias and J. Flores, Phys. Lett. B 35, 383 (1971).

[8] S. S. M. Wong and J. B. French, Nucl. Phys. A 198, 188 (1972).

[9] O. Bohigas, J. Flores, J. B. French, M. J. Giannoni, P. A. Mello, and S. S. M. Wong, Phys. Rev. C 10, 1551 (1974).

[10] H. A. Weidenmüller and G. E. Mitchell, Rev. Mod. Phys. 81, 539 (2009).

[11] S. F. Edwards and R. C. Jones, J. Phys. A: Math. Gen. 9, 1595 (1976).

[12] G. J. Rodgers and A. J. Bray, Phys. Rev. B 37, 3557 (1988).

[13] Y. Kim and A. B. Harris, Phys. Rev. B 31, 7393 (1985).

[14] A. J. Bray and G. J. Rodgers, Phys. Rev. B 38, 11461 (1988).

[15] M. Mezard, G. Parisi, and A. Zee, Nucl. Phys. B 559, 689 (1999).

[16] A. Cavagna, I. Giardina, and G. Parisi, Phys. Rev. Lett. 83, 108 (1999).

[17] A. Cavagna, I. Giardina, and G. Parisi, J. Phys.: Condens. Matter 12, 6295 (2000).

[18] R. Monasson, Eur. Phys. J. B 12, 555 (1999).

[19] G. Biroli and R. Monasson, J. Phys. A: Math. Gen. 32, L255 (1999).

[20] G. Semerjian and L. F. Cugliandolo, J. Phys. A: Math. Gen. 35, 4837 (2002).

[21] D. S. Dean, J. Phys. A: Math. Gen. 35, L153 (2002).

[22] Jean-Yves Fortin, J. Phys. A: Math. Gen. 38, L57 (2005).

[23] G. J. Rodgers, K. Austin, B. Kahng, and D. Kim, J. Phys. A: Math. Gen. 38, 9431 (2005).

[24] G. M. Cicuta and H. Orland, Phys. Rev. E 74, 051120 (2006).

[25] T. Nagao and T. Tanaka, J. Phys. A: Math. Theor. 40, 4973 (2007).

[26] T. Nagao and G. J. Rodgers, J. Phys. A: Math. Theor. 41, 265002 (2008).

[27] R. Kühn, J. Phys. A: Math. Theor. 41, 295002 (2008).

[28] G. Bianconi, arXiv:0804.1744 (2008).

[29] S. N. Evangelou, J. Stat. Phys. 69, 361 (1992).
[30] S. N. Evangelou and E. N. Economou, Phys. Rev. Lett. 68, 361 (1992).

[31] M. Bauer and O. Golinelli, J. Stat. Phys. 103, 301 (2001).

[32] O. Golinelli, cond-mat/0301437

[33] T. Rogers, I. Perez Castillo, R. Kühn, and K. Takeda, Phys. Rev. E 78, 031116 (2008).

[34] P. Cizeau and J. P. Bouchaud, Phys. Rev. E 50, 1810 (1994).

[35] S. N. Dorogovtsev, A. V. Goltsev, J. F. F. Mendes, and A. N. Samukhin, Phys. Rev. E 68, 046109 (2003).

[36] S. N. Dorogovtsev, A. V. Goltsev, J. F. F. Mendes, and A. N. Samukhin, Physica A 338, 76 (2004).

[37] A. N. Samukhin, S. N. Dorogovtsev, and J. F. F. Mendes, Phys. Rev. E 77, 036115 (2008).

[38] C. Bordenave and M. Lelarge, arXiv:0801.0155 (2008).

[39] K. B. Efetov, Adv. Phys. 32, 53 (1983).

[40] J. J. M. Verbaarschot, H. A. Weidenmuller, and M. R. Zirnbauer, Phys. Rep. 129, 367 (1985).

[41] T. Guhr, A. Müller-Groeling, and H. A. Weidenmüller, Phys. Rep. 299, 189 (1998).

[42] J. E. Bunder, K. B. Efetov, V. E. Kravtsov, O. M. Yevtushenko, and M. R. Zirnbauer, J. Stat. Phys. 129, 809 (2007).

[43] J. J. M. Verbaarschot and M. R. Zirnbauer, J. Phys. A: Math. Gen. 18, 1093 (1985).

[44] G. J. Rodgers and C. De Dominicis, J. Phys. A: Math. Gen. 23, 1567 (1990).

[45] Y. V. Fyodorov and A. D. Mirlin, J. Phys. A: Math. Gen. 24, 2219 (1991).

[46] A. D. Mirlin and Y. V. Fyodorov, J. Phys. A: Math. Gen. 24, 2273 (1991).

[47] A. Kamenev and M. Mézard, J. Phys. A: Math. Gen. 32, 4373 (1999).

[48] E. Kanzieper, Nucl. Phys. B 596, 548 (2001).

[49] E. Kanzieper, arXiv:0909.3198, to appear in: The Oxford Handbook of Random Matrix Theory (2009).

[50] V. A. Osipov and E. Kanzieper, Phys. Rev. Lett. 99, 050602 (2007).

[51] A. Khorunzhy and G. J. Rodgers, J. Math. Phys. 38, 3300 (1997).

[52] P.-O. Löwdin, J. Appl. Phys. 33, 251 (1962).

[53] Z. Füredi and J. Komlós, Combinatorica 1, 233 (1981).

[54] V. A. Marčenko and L. A. Pastur, Math. USSR-Sbornik 1, 457 (1967). 